\title{
Atributos químicos e área superficial específica em Latossolo subtropical de altitude sob usos e manejos distintos
}

\author{
Chemical attributes and specific surface area in highland subtropical Oxisol under different use and \\ managements
}

\author{
Cristiano Albino Tomasi ${ }^{\mathrm{I}}$ Alberto Vasconcellos Inda ${ }^{\mathrm{I}^{*}}$ Deborah Pinheiro Dick $^{\mathrm{II}}$ \\ Carlos Alberto Bissani ${ }^{I}$ Jessé Rodrigo Fink ${ }^{\mathrm{I}}$
}

\section{RESUMO}

\begin{abstract}
A interferência antrópica tem modificado a condição original do solo nos Campos de Cima da Serra. O estudo avaliou atributos químicos e físicos de um Latossolo sob campo nativo $(C N)$, campo nativo manejado com queima $(C N q)$, mata nativa $(M N)$, florestamento de pinus $(F P)$ e lavoura em sistema plantio convencional (LA), nas camadas de 0,00$0,025,0,025-0,05,0,05-0,10,0,10-0,20,0,20-0,30 m$. A mineralogia foi avaliada por difratometria de raios $X$. Foram avaliados o C orgânico total (COT), $\mathrm{pH}\left(\mathrm{H}_{2} \mathrm{O}\right) ; \mathrm{Ca}, \mathrm{Mg}, \mathrm{K}, \mathrm{Na}$ e Al trocáveis; $\mathrm{H}+\mathrm{Al}$ e $\mathrm{P}$; e calculou-se a soma de bases, a capacidade de troca de cátions, a saturação por bases e por Al. Estimou-se a área superficial específica (ASE) e a capacidade máxima de adsorção de fósforo (CMAP). O solo apresentou mineralogia caulinítica e oxídica. No CN, o COT variou entre 15,3 e 56,4 $\mathrm{kg}^{-1}$, o $\mathrm{pH}$ foi $\leq 4,8$, a CTC foi alta $\left(18<\mathrm{CTC}_{p H 7}<22 \mathrm{cmol}_{c} \mathrm{dm}^{-3}\right)$, com baixa saturação por bases $(3<V<34 \%)$ e alta saturação por Al $(23<m<88 \%)$. Em comparação ao CN, o COT aumentou em superfície na $M N$, foi equivalente no $\mathrm{CNq}$ e diminuiu sob FP e LA. A fertilidade do solo aumentou sob $M N$ e CNq, e foi reduzida sob FP, tendo sido condicionada pelo teor de COT. O COT mostrou relação positiva com a ASE e negativa com a CMAP do solo.
\end{abstract}

Palavras-chave: adsorção de fósforo, queimada, pinus.

\begin{abstract}
The original landscape of Campos de Cima da Serra region has changed by anthropogenic interference. The study aimed to evaluate soil chemical and physical attributes of an Oxisol under natural grassland $(C N)$, burned natural grassland $(C N q)$, natural forest $(M N)$, pine afforestation $(P F)$ and annual crops in conventional tillage system $(L A)$, in the $0.00-0.025,0.025-0.05,0.05-0.10,0.10-0.20,0.20-0.30 \mathrm{~m}$
\end{abstract}

\begin{abstract}
layers. The mineralogy was evaluated by $X$-ray diffraction. It was evaluated the total organic carbon (TOC); $\mathrm{pH}\left(\mathrm{H}_{2} \mathrm{O}\right)$; exchangeable $\mathrm{Ca}, \mathrm{Mg}, \mathrm{K}, \mathrm{Na}$ and $\mathrm{Al} ; \mathrm{H}+\mathrm{Al}$ and $\mathrm{P}$ contents; and calculated bases sum (S), cation exchange capacity (CEC) and bases and Al saturation on CEC. It was estimated soil specific surface area (SSA) and maximum phosphorus adsorption capacity (MPAC). The soil showed kaolinitic and oxidic mineralogy. In the $C N$ soil TOC content ranged between 15.3 and $56.4 \mathrm{~g} \mathrm{~kg}^{-1}$, pH values $\leq 4.8$; CEC was high $\left(18<\mathrm{CEC}_{p H 7}<22 \mathrm{cmol}_{c} \mathrm{dm}^{-3}\right)$ with low bases saturation $(3<V<34 \%)$ and high Al saturation $(23<m<88 \%)$. Related to $C N$ soil, TOC contents increased in the MN surface was similar for the CNq and decreased for the FP and LA areas. Soil fertility was positively modified in $M N$ and $C N Q$ areas, and reduced under FP, and was conditioned by TOC. The TOC content was positively related to soil ASE and negatively with soil MPAC.
\end{abstract}

Key words: phosphorus adsorption, burning, pinus.

\section{INTRODUÇÃO}

A região dos Campos de Cima da Serra (CCS), situada na porção nordeste do Rio Grande do Sul, caracteriza-se por condições que determinam, originalmente, uma situação rara no mundo, de cobertura vegetal herbácea entremeada por matas nativas de araucárias (OVERBECK et al., 2007). Historicamente, o uso da terra com pastagem nativa tem sido a principal atividade econômica nessa região, associada a uma prática antiga de manejo dessas pastagens, que é a queima no final do inverno, visando

'Departamento de Solos, Faculdade de Agronomia, Universidade Federal do Rio Grande do Sul (UFRGS), 91540-000, Porto

Alegre, RS, Brasil. E-mail: alberto.inda@ufrgs.br.*Autor para correspondência.

IIDepartamento de Físico-Química, Instituto de Química, UFRGS, Porto Alegre, RS, Brasil. 
a eliminar as sobras de pasto envelhecidas e facilitar o rebrote da vegetação(HERINGER \& JACQUES, 2002a). Mais recentemente, uma alternativa ao regime de queima tem sido o melhoramento da pastagem nativa, através da aplicação de calcário e fertilizantes, da utilização de roçada e da introdução de novas espécies para suprir as deficiências de forragem no inverno (HERINGER \& JACQUES, 2002a, b).

A expansão de atividades agropecuárias tem modificado mais intensamente a formação original na região dos CCS nas últimas três décadas (OVERBECK et al., 2007), com destaque para a conversão de áreas originalmente de campos, usadas com pastagem, em lavouras de culturas anuais (ex. Zea mays, Glycine max, e Triticum aestivum) e em florestas com espécies exóticas (principalmente Pinus spp.).

Nesta região, tais mudanças no uso do solo alteram quantitativa e qualitativamente a dinâmica da matéria orgânica do solo (DICK et al., 2008; MAFRA et al., 2008; WIESMEIER et al., 2009; DICK et al., 2011), com efeitos importantes nos atributos químicos dos solos (HERINGER et al., 2002; RHEINHEIMER, et al., 2003; DICK et al., 2008; DICK et al., 2011), devido à baixa reserva nutricional nos solos altamente intemperizados dos CCS (STRECK et al., 2008).

Considerando a diversidade de classes de solo na região dos CCS e a demanda em compreender o comportamento desses solos frente a novos usos, este estudo teve como objetivo investigar os impactos de diferentes usos e manejos nos teores de carbono orgânico e nos atributos químicos e físico de um Latossolo na região dos CCS.

\section{MATERIAL E MÉTODOS}

O estudo foi realizado no município de André da Rocha ( $28^{\circ} 38^{\prime}$ 'S, 51 $34^{\prime}$ 'O), no Rio Grande do Sul. O clima é temperado úmido $(\mathrm{Cfb})$ com uma temperatura média anual de $17,6^{\circ} \mathrm{C}$ e uma precipitação média anual entre 1600 e $1700 \mathrm{~mm}$. O solo é classificado como Latossolo Vermelho distroférrico (STRECK et al., 2008). A vegetação natural representa uma transição entre campo e mata, com dominância de gramíneas cespitosas.

O solo foi avaliado sob os seguintes usos e manejo, respectivamente: campo nativo pastejado sem queima e sem roçada há 43 anos $(\mathrm{CN})$; mata nativa $(\mathrm{MN})$; lavoura há 40 anos, sob sistema de preparo convencional - SPC, com sucessão anual soja/aveia/ azevém/milho, e calagem a cada quatro anos (LA); florestamento com Pinus elliottis, estabelecido há 18 anos sobre campo nativo manejado com queima pós inverno (FP); e campo nativo pastejado e manejado com queima cada ano após o inverno há mais de 100 anos $(\mathrm{CNq})$. $\mathrm{O}$ uso $\mathrm{CN}$ foi utilizado como referencial para avaliação das alterações dos atributos químicos e físico do solo, provocadas pelos demais usos (MN; LA; FP) e manejo $(\mathrm{CNq})$ do solo. A área de cada tratamento foi de $50 \times 50 \mathrm{~m}$, totalizando $12.500 \mathrm{~m}^{2}$. O delineamento experimental utilizado foi o inteiramente casualizado, com três repetições. Em cada tratamento, foram abertas três trincheiras e coletadas amostras de solo nas camadas de 0,00-0,025, 0,025-0,05, 0,05-0,10, $0,10-0,20,0,20-0,30 \mathrm{~m}$. As amostras foram destorroadas, secas ao ar, moídas e peneiradas em malha de $2 \mathrm{~mm}$ para obtenção da fração TFSA. Em amostra da TFSA sem purificação, a fração argila total do solo foi obtida por sedimentação, segundo a Lei de Stokes, após a dispersão total das partículas por ultrassom.

As análises químicas foram realizadas na fração TFSA, segundo TEDESCO et al. (1995), para os seguintes componentes: $\mathrm{pH}$ em $\mathrm{H}_{2} \mathrm{O}, \mathrm{Ca}, \mathrm{Mg}, \mathrm{K}, \mathrm{Na}$, $\mathrm{Al}, \mathrm{H}+\mathrm{Al}$ e P. Foram calculadas a soma de bases (S), a capacidade de troca de cátions $\left(\mathrm{CTC}_{\mathrm{pH}}\right)$, a saturação por bases (V) e a saturação por $\mathrm{Al}(\mathrm{m})$. A determinação do Corgânico total (COT) foi realizada por combustão seca em analisador de carbono SHIMADZU TOC-V.

Para a avaliação da adsorção de $P$, utilizaramse $3 \mathrm{~g}$ de amostra de TFSA da camada $0,00-0,05 \mathrm{~m}$, que foi equilibrada com $30 \mathrm{~mL}$ de $\mathrm{KCl} 0,02 \mathrm{~mol} \mathrm{~L}^{-1}$ contendo doses de 30, 60, 120, 240, 480, 960, 1.920 $\mathrm{mg} \mathrm{dm}^{-3} \mathrm{de}$. As suspensões foram agitadas por 5 horas e deixadas em repouso por 20 horas. $\mathrm{O}$ teor de $\mathrm{P}$ no sobrenadante foi determinado de acordo com o método MURPHY \& HILEY (1962). O P adsorvido foi estimado pela diferença entre o P adicionado e o P do sobrenadante. A capacidade máxima de adsorção de fósforo (CMAP) foi estimada através da equação de Langmuir ajustada $\left[\mathrm{P}_{\text {adsorvido }}=\mathrm{KP}_{\text {max }} \mathrm{P}_{\text {solução }} /\left(1+\mathrm{KP}_{\text {solução }}\right]\right.$, em que $\mathrm{P}_{\text {adsorvido }}$ é o $\mathrm{P}$ adsorvido na amostra, $\mathrm{P}_{\text {solução }}$ é a concentração na solução, Ké a constante referente à energia de sorção, $\mathrm{P}_{\max }$ é a capacidade máxima de adsorção $\mathrm{P}$.

A área superficial específica (ASE) da TFSA da camada 0,00-0,05m foi estimada por adsorção de água em atmosfera de 20\% de umidade (QUIRK, 1955). A mineralogia foi analisada por difratometria de raios $\mathrm{X}$ (DRX) (Difratômetro Siemens D5000, radiação de $\mathrm{CuK} \alpha$ e filtro de $\mathrm{Ni}$, voltagem de $40 \mathrm{kV}$ e corrente de $25 \mathrm{~mA}$ ) em amostra da camada 0,00-0,20m do solo sob $\mathrm{CN}$. As análises foram efetuadas em lâminas não orientadas da fração argila desferrificada com ditionitocitrato-bicarbonato de sódio (MEHRA \& JACKSON, 1960), no intervalo de 2 a $72^{\circ} 2 \theta$; e da fração óxidos de ferro concentrada após tratamento da fração argila total com $\mathrm{NaOH} 5 \mathrm{~mol} \mathrm{~L}^{-1}$ fervente (KÄMPF \& SCHWERTMANN, 1982), no intervalo de 15 a $50^{\circ} 2 \theta$. 
Análises de correlação entre os parâmetros avaliados e a comparação de médias pelo teste de Tukey em nível de 5\% de probabilidade foram realizadas com o auxílio do programa estatístico SAS for Windows 9.0.

\section{RESULTADOS E DISCUSSÃO}

Mineralogia do solo

A composição mineralógica da fração argila apresentou, com base na intensidade das reflexões nos DRX (difratogramas não mostrados), uma dominância de caulinita associada a quartzo e cristobalita. Na fração óxidos de ferro concentrada, foram identificados os óxidos de ferro goethita e hematita, com pequena predominância do primeiro $(\mathrm{Hm} /(\mathrm{Hm}+\mathrm{Gt})=0,40)$.

\section{Carbono orgânico total}

$\mathrm{O} \mathrm{CN}$ foi considerado o ambiente de referência, pois a expansão das florestas sobre os campos é um processo relativamente recente (1.100 anos) (OVERBECK et al., 2007). No CN, o COT foi alto em superfície e decresceu com a profundidade (Tabela 1). Os teores atuais de $\mathrm{C}$ no $\mathrm{CN}$ são resultado do aporte de resíduos vegetais associado a uma lenta taxa de degradação, determinada pelo clima nos CCS (OVERBECK et al., 2007). Em comparação ao CN, os teores de COT na MN foram maiores na camada 0,00 $0,05 \mathrm{~m}$, possivelmente pela associação de plantas rasteiras e arbóreas, a qual determina um maior aporte de resíduos vegetais.

No CNq, o manejo do solo por meio da queima da vegetação não afetou os teores de COT (Tabela 1). OVERBECK et al. (2007) indicaram que a queima superficial do $\mathrm{CN}$ teve impacto somente sobre a qualidade das forragens, verificado a médio e longo prazo, pela infestação de espécies invasoras, por perdas do valor nutritivo (HERINGER \& JACQUES, 2002b) e pela baixa diversidade de espécies vegetais (PILLAR \& QUADROS, 1997). Exceto na camada mais superficial $(0,00-0,025 \mathrm{~m})$, a LA e o FP diminuíram os teores de COT em relação ao ambiente $\mathrm{CN}$ (Tabela 1). $\mathrm{Na} \mathrm{LA}$, a redução de COT se deve à condição do solo mais oxidativa, causada pelo revolvimento anual da camada superficial do solo no SPC. No FP, outros estudos também têm constatado depleções de COT (WIESMEIER et al., 2009; DICK et al., 2011), as quais foram relacionadas à baixa taxa de decomposição das acículas do pinus, decorrentes do alto teor de lignina e de compostos fenólicos de sua estrutura (CARVALHO et al., 2008; WIESMEIER et al., 2009).

\section{Atributos químicos}

No $\mathrm{CN}$, o pH variou entre 4,6 e 4,8 e a capacidade de troca de cátions foi alta, variando de 18 a $22 \mathrm{cmol}_{\mathrm{c}} \mathrm{dm}^{-3}$ (Tabelas $2 \mathrm{e} 3$ ). A dessaturação do solo se expressou na baixa soma de bases $(0,8 \leq \mathrm{S} \leq 6,3 \mathrm{cmolc}$ $\left.\mathrm{dm}^{-3}\right)$ e saturação por bases $(3<\mathrm{V}<34 \%)$, as quais, entretanto, aumentaram em superfície devido a biociclagem. $\mathrm{O} \mathrm{Al}$ trocável foi alto $(1,9 \leq \mathrm{Al} \leq 5,5 \mathrm{cmolc}$ $\left.\mathrm{dm}^{-3}\right)$, bem como a saturação por $\mathrm{Al}(23<\mathrm{m}<88 \%)$. O P disponível variou de médio $\left(4,1-6,0 \mathrm{mg} \mathrm{dm}^{-3}\right)$ em superfície a baixo $\left(2,1-4,0 \mathrm{mg} \mathrm{dm}^{-3}\right)$ com o aumento da profundidade, reforçando o efeito da biociclagem. Estudos em solos sob CN nessa região descrevem resultados semelhantes (HERINGER et al., 2002; DICK et al., 2008) que expressam a avançada intemperização desses solos, condizente com a composição mineralógica, caulinítica e oxídica.

$\mathrm{Na} \mathrm{MN}$, os níveis de fertilidade foram melhores e a CTC do solo maior que no $\mathrm{CN}$, principalmente em superfície (Tabelas 2 e 3 ). $\mathrm{O} \mathrm{Al}$ trocável e a saturação por $\mathrm{Al}$ foram menores em

Tabela 1 - Carbono orgânico total (COT) em diferentes camadas de um Latossolo sob campo nativo (CN), campo nativo queimado (CNq), lavoura (LA), florestamento de pinus (FP) e mata nativa (MN).

\begin{tabular}{llccccc}
\hline & & & & & \\
& Ambiente & & & & \\
& & $0,00-0,025$ & $0,025-0,05$ & $0,05-0,10$ & $0,10-0,20$ & $0,20-0,30$ \\
\hline & CN & $56,4 \mathrm{~b}$ & $40,7 \mathrm{~b}$ & $31,7 \mathrm{a}$ & $28,1 \mathrm{a}$ & $25,3 \mathrm{a}$ \\
$\mathrm{COT}, \mathrm{g} \mathrm{kg}^{-1}$ & $\mathrm{CNq}$ & $55,5 \mathrm{~b}$ & $40,5 \mathrm{~b}$ & $33,3 \mathrm{a}$ & $27,3 \mathrm{a}$ & $22,4 \mathrm{ab}$ \\
& $\mathrm{LA}$ & $28,7 \mathrm{~b}$ & $24,6 \mathrm{c}$ & $22,4 \mathrm{~b}$ & $20,4 \mathrm{~b}$ & $18,1 \mathrm{~b}$ \\
& FP & $31,9 \mathrm{~b}$ & $22,2 \mathrm{c}$ & $21,3 \mathrm{~b}$ & $21,5 \mathrm{~b}$ & $18,9 \mathrm{~b}$ \\
& MN & $106,4 \mathrm{a}$ & $58,1 \mathrm{a}$ & $35,2 \mathrm{a}$ & $26,6 \mathrm{a}$ & $20,8 \mathrm{ab}$ \\
& CV\% & 22,7 & 10,0 & 6,3 & 7,0 & 8,7 \\
\hline
\end{tabular}

Médias seguidas da mesma letra em cada coluna não diferem pelo teste Tukey $(\mathrm{P}<0,05)$.

Ciência Rural, v.42, n.12, dez, 2012. 
Tabela 2 - Valores de pH em água e teores de elementos químicos em diferentes camadas de um Latossolo sob campo nativo (CN), campo nativo queimado $(\mathrm{CNq})$, lavoura (LA), florestamento de pinus (FP) e mata nativa (MN).

\begin{tabular}{|c|c|c|c|c|c|c|}
\hline \multirow{2}{*}{ Atributo químico } & \multirow{2}{*}{ Ambiente } & \multicolumn{5}{|c|}{-Profundidade (m)--- } \\
\hline & & $0,00-0,025$ & $0,025-0,05$ & $0,05-0,10$ & $0,10-0,20$ & $0,20-0,30$ \\
\hline \multirow{6}{*}{$\mathrm{pH} \mathrm{em} \mathrm{H}_{2} \mathrm{O}$} & $\mathrm{CN}$ & $4,8 \mathrm{bc}$ & $4,7 \mathrm{c}$ & $4,7 \mathrm{c}$ & $4,7 \mathrm{bc}$ & $4,6 \mathrm{bc}$ \\
\hline & $\mathrm{CNq}$ & $5,8 \mathrm{a}$ & $5,3 \mathrm{~b}$ & $5,1 \mathrm{~b}$ & $5,0 \mathrm{~b}$ & $5,0 \mathrm{~b}$ \\
\hline & LA & $5,6 \mathrm{a}$ & $5,6 \mathrm{a}$ & $5,8 \mathrm{a}$ & $5,8 \mathrm{a}$ & $5,6 \mathrm{a}$ \\
\hline & FP & $4,5 \mathrm{c}$ & $4,6 \mathrm{c}$ & $4,5 \mathrm{c}$ & $4,5 \mathrm{c}$ & $4,6 \mathrm{bc}$ \\
\hline & MN & $5,1 \mathrm{~b}$ & $4,8 \mathrm{c}$ & $4,7 \mathrm{c}$ & $4,6 \mathrm{c}$ & $4,5 \mathrm{c}$ \\
\hline & $\mathrm{CV} \%$ & 3,5 & 1,9 & 2,8 & 2,6 & 2,3 \\
\hline \multirow{6}{*}{$\mathrm{K}, \mathrm{cmol}_{\mathrm{c}} \cdot \mathrm{dm}^{-3}$} & $\mathrm{CN}$ & $0,94 \mathrm{a}$ & $0,79 \mathrm{ab}$ & $0,63 \mathrm{a}$ & $0,49 \mathrm{a}$ & $0,31 \mathrm{a}$ \\
\hline & $\mathrm{CNq}$ & $1,02 \mathrm{a}$ & $1,02 \mathrm{a}$ & $0,72 \mathrm{a}$ & $0,42 \mathrm{a}$ & $0,23 \mathrm{a}$ \\
\hline & LA & $0,32 \mathrm{c}$ & $0,17 \mathrm{c}$ & $0,10 \mathrm{~b}$ & $0,07 \mathrm{~b}$ & $0,05 \mathrm{a}$ \\
\hline & FP & $0,09 \mathrm{~d}$ & $0,07 \mathrm{c}$ & $0,07 \mathrm{~b}$ & $0,06 \mathrm{~b}$ & $0,04 \mathrm{a}$ \\
\hline & MN & $0,71 \mathrm{~b}$ & $0,58 \mathrm{~b}$ & $0,40 \mathrm{ab}$ & $0,29 \mathrm{ab}$ & $0,27 \mathrm{a}$ \\
\hline & $\mathrm{CV} \%$ & 13,1 & 26,8 & 34,9 & 44,8 & 56,8 \\
\hline \multirow{6}{*}{$\mathrm{Ca}, \mathrm{cmol}_{\mathrm{c}} \cdot \mathrm{dm}^{-3}$} & $\mathrm{CN}$ & $3,0 \mathrm{~b}$ & $2,4 a b$ & $1,3 \mathrm{a}$ & $1,1 \mathrm{~b}$ & $0,7 \mathrm{~b}$ \\
\hline & $\mathrm{CNq}$ & $6,6 \mathrm{~b}$ & $3,6 \mathrm{ab}$ & $2,4 \mathrm{a}$ & $1,7 \mathrm{~b}$ & $1,2 \mathrm{~b}$ \\
\hline & LA & $7,0 \mathrm{~b}$ & $6,9 \mathrm{ab}$ & $7,7 \mathrm{a}$ & $7,6 \mathrm{a}$ & $4,6 \mathrm{a}$ \\
\hline & FP & $1,4 \mathrm{~b}$ & $0,7 \mathrm{~b}$ & $0,5 \mathrm{a}$ & $0,5 \mathrm{~b}$ & $0,3 \mathrm{~b}$ \\
\hline & $\mathrm{MN}$ & $17,9 \mathrm{a}$ & $11,0 \mathrm{a}$ & $5,3 \mathrm{a}$ & $1,3 \mathrm{~b}$ & $0,5 \mathrm{~b}$ \\
\hline & $\mathrm{CV} \%$ & 44,5 & 68,4 & 77,9 & 24,3 & 46,8 \\
\hline \multirow{6}{*}{$\mathrm{Mg}, \mathrm{cmol}_{\mathrm{c}} \cdot \mathrm{dm}^{-3}$} & $\mathrm{CN}$ & $2,3 \mathrm{bc}$ & $2,0 \mathrm{~b}$ & $1,0 \mathrm{~b}$ & $0,8 \mathrm{bc}$ & $0,4 \mathrm{~b}$ \\
\hline & $\mathrm{CNq}$ & $4,6 \mathrm{a}$ & $2,7 \mathrm{ab}$ & $1,7 \mathrm{~b}$ & $1,1 \mathrm{~b}$ & $0,7 \mathrm{~b}$ \\
\hline & LA & $4,1 \mathrm{ab}$ & $3,7 \mathrm{a}$ & $3,9 \mathrm{a}$ & $3,7 \mathrm{a}$ & $2,8 \mathrm{a}$ \\
\hline & FP & $0,8 \mathrm{c}$ & $0,5 \mathrm{c}$ & $0,4 \mathrm{~b}$ & $0,3 \mathrm{c}$ & $0,2 \mathrm{~b}$ \\
\hline & $\mathrm{MN}$ & $4,9 \mathrm{a}$ & $3,5 \mathrm{a}$ & $2,0 \mathrm{~b}$ & $1,1 \mathrm{~b}$ & $0,6 \mathrm{~b}$ \\
\hline & $\mathrm{CV} \%$ & 20,5 & 19,2 & 32,7 & 19,8 & 40,4 \\
\hline \multirow{6}{*}{$\mathrm{S}, \mathrm{cmol}_{\mathrm{c} \cdot} \cdot \mathrm{dm}^{-3}$} & $\mathrm{CN}$ & $6,3 \mathrm{bc}$ & $5,2 \mathrm{ab}$ & $3,0 \mathrm{~b}$ & $2,4 \mathrm{bc}$ & $1,5 \mathrm{~b}$ \\
\hline & $\mathrm{CNq}$ & $12,3 \mathrm{~b}$ & $7,5 \mathrm{ab}$ & $4,9 \mathrm{ab}$ & $3,3 \mathrm{~b}$ & $2,3 \mathrm{~b}$ \\
\hline & LA & $11,5 \mathrm{bc}$ & $10,9 \mathrm{ab}$ & $11,7 \mathrm{a}$ & $11,4 \mathrm{a}$ & $7,5 \mathrm{a}$ \\
\hline & FP & $2,47 \mathrm{~d}$ & $1,3 \mathrm{~b}$ & $1,1 \mathrm{~b}$ & $0,9 \mathrm{c}$ & $0,6 \mathrm{~b}$ \\
\hline & $\mathrm{MN}$ & $23,5 \mathrm{a}$ & $15,1 \mathrm{a}$ & $7,7 \mathrm{ab}$ & $2,7 \mathrm{bc}$ & $1,4 \mathrm{~b}$ \\
\hline & $\mathrm{CV} \%$ & 30,9 & 46,1 & 55,1 & 19,8 & 41,0 \\
\hline \multirow{6}{*}{$\mathrm{Al}, \mathrm{cmol}_{\mathrm{c}} \cdot \mathrm{dm}^{-3}$} & $\mathrm{CN}$ & $1,9 \mathrm{~b}$ & $3,1 \mathrm{~b}$ & $4,1 \mathrm{ab}$ & $4,7 \mathrm{a}$ & $5,2 \mathrm{a}$ \\
\hline & $\mathrm{CNq}$ & $0,1 \mathrm{c}$ & $1,0 \mathrm{c}$ & $1,6 \mathrm{bc}$ & $2,3 \mathrm{~b}$ & $2,5 \mathrm{~b}$ \\
\hline & LA & $0,0 \mathrm{c}$ & $0,3 \mathrm{c}$ & $0,0 \mathrm{c}$ & $0,0 \mathrm{c}$ & $0,5 \mathrm{c}$ \\
\hline & FP & $5,3 \mathrm{a}$ & $5,2 \mathrm{a}$ & $5,5 \mathrm{a}$ & $5,7 \mathrm{a}$ & $5,0 \mathrm{a}$ \\
\hline & $\mathrm{MN}$ & $0,2 \mathrm{c}$ & $1,0 \mathrm{c}$ & $2,5 \mathrm{bc}$ & $5,2 \mathrm{a}$ & $5,7 \mathrm{a}$ \\
\hline & $\mathrm{CV} \%$ & 24,3 & 29,9 & 38,4 & 15,5 & 9,8 \\
\hline \multirow{6}{*}{$\mathrm{P}, \mathrm{mg} \mathrm{dm}^{-3}$} & $\mathrm{CN}$ & $5,6 \mathrm{~b}$ & $4,0 \mathrm{~b}$ & $3,7 \mathrm{a}$ & $2,9 \mathrm{a}$ & $2,2 \mathrm{ab}$ \\
\hline & $\mathrm{CNq}$ & $6,7 \mathrm{~b}$ & $3,4 \mathrm{~b}$ & $2,9 \mathrm{a}$ & $2,5 \mathrm{a}$ & $2,2 \mathrm{ab}$ \\
\hline & LA & $18,0 \mathrm{a}$ & $15,5 \mathrm{a}$ & $10,4 \mathrm{a}$ & $3,7 \mathrm{a}$ & $2,5 \mathrm{a}$ \\
\hline & $\mathrm{FP}$ & $3,7 \mathrm{~b}$ & $3,0 \mathrm{~b}$ & $3,0 \mathrm{a}$ & $2,3 \mathrm{a}$ & $2,0 \mathrm{~b}$ \\
\hline & $\mathrm{MN}$ & $10,5 \mathrm{~b}$ & $7,1 \mathrm{~b}$ & $5,4 \mathrm{a}$ & $3,1 \mathrm{a}$ & $2,6 \mathrm{a}$ \\
\hline & $\mathrm{CV} \%$ & 29,4 & 42,6 & 56,2 & 19,8 & 8,9 \\
\hline
\end{tabular}

K: potássio; Ca: cálcio; Mg: magnésio; S: soma de bases; Al: alumínio; P: fósforo; CV: Coeficiente de Variação (\%). Médias seguidas da mesma letra em cada coluna não diferem pelo teste Tukey $(\mathrm{P}<0,05)$. 
Tabela 3 - Capacidade de troca de cátions (CTC), saturação por bases (V) e saturação por alumínio (m) em diferentes camadas de um Latossolo sob campo nativo $(\mathrm{CN})$, campo nativo queimado $(\mathrm{CNq})$, lavoura (LA), florestamento de pinus (FP) e mata nativa (MN).

\begin{tabular}{|c|c|c|c|c|c|c|}
\hline \multirow{2}{*}{ Atributo químico } & \multirow{2}{*}{ Ambiente } & \multicolumn{5}{|c|}{-Profundidade $(\mathrm{m})$-- } \\
\hline & & $0,00-0,025$ & $0,025-0,05$ & $0,05-0,10$ & $0,10-0,20$ & $0,20-0,30$ \\
\hline \multirow{6}{*}{ CTC, $\mathrm{cmol}_{\mathrm{c}} \cdot \mathrm{dm}^{-3}$} & $\mathrm{CN}$ & $18,6 \mathrm{c}$ & $20,8 \mathrm{ab}$ & $20,2 a b$ & $21,3 a b$ & $21,7 \mathrm{a}$ \\
\hline & $\mathrm{CNq}$ & $18,4 \mathrm{c}$ & $14,8 \mathrm{c}$ & $13,4 \mathrm{c}$ & $12,6 \mathrm{~b}$ & $12,8 \mathrm{~b}$ \\
\hline & LA & $16,8 \mathrm{c}$ & $16,5 \mathrm{bc}$ & $15,8 \mathrm{bc}$ & $15,2 \mathrm{ab}$ & $11,5 \mathrm{~b}$ \\
\hline & FP & $25,1 \mathrm{~b}$ & $23,9 \mathrm{a}$ & $23,7 \mathrm{a}$ & 22,9 a & $21,6 \mathrm{a}$ \\
\hline & $\mathrm{MN}$ & $30,4 \mathrm{a}$ & $24,0 \mathrm{a}$ & $19,7 \mathrm{ab}$ & $24,5 \mathrm{a}$ & $23,5 \mathrm{a}$ \\
\hline & $\mathrm{CV} \%$ & 8,4 & 8,1 & 10,3 & 19,0 & 13,7 \\
\hline \multirow{6}{*}{$\mathrm{V}, \%$} & $\mathrm{CN}$ & $34,0 \mathrm{~b}$ & $25,5 \mathrm{bc}$ & $16,1 \mathrm{~b}$ & $11,9 \mathrm{c}$ & $6,8 \mathrm{bc}$ \\
\hline & $\mathrm{CNq}$ & $66,7 \mathrm{a}$ & $50,3 \mathrm{ab}$ & $37,2 \mathrm{ab}$ & $25,9 \mathrm{~b}$ & $17,8 \mathrm{~b}$ \\
\hline & LA & $68,4 \mathrm{a}$ & $66,1 \mathrm{a}$ & 73,8 a & $75,1 \mathrm{a}$ & $64,0 \mathrm{a}$ \\
\hline & FP & $9,5 \mathrm{~b}$ & $5,5 \mathrm{c}$ & $4,5 \mathrm{~b}$ & $4,1 \mathrm{c}$ & $3,0 \mathrm{c}$ \\
\hline & MN & $76,4 \mathrm{a}$ & $61,4 \mathrm{ab}$ & $40,6 \mathrm{ab}$ & $12,3 \mathrm{c}$ & $6,1 \mathrm{bc}$ \\
\hline & $\mathrm{CV} \%$ & 18,4 & 32,3 & 51,3 & 17,6 & 27,4 \\
\hline \multirow{6}{*}{$\mathrm{m}, \%$} & $\mathrm{CN}$ & $23,4 \mathrm{~b}$ & $37,8 \mathrm{~b}$ & $58,8 \mathrm{ab}$ & $66,0 \mathrm{a}$ & $77,9 \mathrm{a}$ \\
\hline & $\mathrm{CNq}$ & $1,1 \mathrm{c}$ & $11,9 \mathrm{c}$ & $25,3 \mathrm{bc}$ & $41,8 \mathrm{~b}$ & $54,0 \mathrm{~b}$ \\
\hline & LA & $0,6 \mathrm{c}$ & $3,3 \mathrm{c}$ & $0,0 \mathrm{c}$ & $0,0 \mathrm{c}$ & $7,1 \mathrm{c}$ \\
\hline & FP & $69,2 \mathrm{a}$ & $79,8 \mathrm{a}$ & $84,1 \mathrm{a}$ & 86,0 a & 88,6 a \\
\hline & MN & $1,5 \mathrm{c}$ & $8,4 \mathrm{c}$ & $33,0 \mathrm{bc}$ & $66,1 \mathrm{a}$ & $80,3 \mathrm{a}$ \\
\hline & $\mathrm{CV} \%$ & 15,7 & 24,7 & 38,4 & 14,5 & 13,6 \\
\hline
\end{tabular}

CV: Coeficiente de Variação (\%). Médias seguidas da mesma letra em cada coluna não diferem pelo teste Tukey $(\mathrm{P}<0,05)$.

comparação ao CN na camada 0,00-0,05m. Também nas camadas superficiais, foram maiores os teores de $\mathrm{Ca}$, $\mathrm{Mg}$ e, consequentemente, da soma de bases e da saturação por bases, sugerindo uma biociclagem mais intensa sob MN, reforçada pela tendência de aumento do $\mathrm{P}$ disponível, não confirmada estatisticamente devido aos altos coeficientes de variação para este e outros atributos avaliados. Os menores teores de $\mathrm{K} \mathrm{em}$ superfície, comparativamente ao $\mathrm{CN}$, confirmam estudo realizado por Mafra et al. (2008), que indicou redução desse elemento em solo sob vegetação arbórea.

A queima dos resíduos vegetais anualmente por mais de 100 anos no $\mathrm{CNq}$ favoreceu o aumentou do $\mathrm{pH}$ e da concentração de cátions básicos na camada 0,00-0,10m, conforme indicaram os teores de $\mathrm{Mg}$ e a saturação por bases (Tabelas 2 e 3). O Al trocável e a saturação por $\mathrm{Al}$ foram reduzidos, contrastando com estudos anteriores que indicam alterações negativas na fertilidade de solos sob regime de queima anual da pastagem (HERINGER et al., 2002; DICK et al., 2008). Neste estudo, a amostragem no $\mathrm{CNq}$ foi realizada somente sete dias após a queima e, nesse curto prazo, o efeito da formação de cinzas com altas concentrações de cátions básicos e $\mathrm{P}$ pode ter intensificado a velocidade de mineralização desses elementos (RHEINHEIMER, et al., 2003). Entretanto, os benefícios do manejo dos campos com o uso da queima, verificados no CNq, têm se mostrado efêmeros e mais restritos à camada $0-0,05 \mathrm{~m}$, com retorno às condições originais ou inferiores, a partir de 90 dias após a queima (RHEINHEIMER, et al., 2003; DICK, et al., 2008).

$\mathrm{O}$ FP alterou negativamente os atributos químicos do solo em comparação ao CN (Tabelas 2 e 3). $\mathrm{OpH}$ indicou tendência de acidificação, sendo, em média, 0,2 unidades inferior ao do $\mathrm{CN}$, similar ao verificado em FP com idades entre 8 e 30 anos (DICK et al., 2011; MAFRA et al., 2008). A acidificação e a redução de bases em FP nos CCS decorrem da intensa lixiviação, da absorção de cátions pelo pinus e da liberação de ácidos orgânicos pela decomposição das acículas (MAFRA et al., 2008). De maneira inversa, o FP aumentou o Al trocável e a saturação por Al nas camadas mais superficiais do solo. Ao contrário dos demais atributos químicos, os teores de $\mathrm{P}$ disponíveis foram similares nos solos sob CN e FP, concordando com a baixa extração desse elemento do solo, conforme a escala total de retiradas de elementos pela cultura do pinus (LA TORRACA, 1984).

$\mathrm{Na}$ LA, as alterações nos atributos químicos do solo se devem à correção da acidez com calcário e à fertilização do solo nos cultivos de verão (milho/soja) e inverno (aveia e/ou azevém) (Tabelas 2 e 3). $\mathrm{O}$ pH 
aumentou para níveis superiores a 5,3, bem como os teores de $\mathrm{Ca}$ e $\mathrm{Mg}$, a soma de bases $\left(>10 \mathrm{cmol}_{\mathrm{c}} \mathrm{dm}^{-3}\right) \mathrm{e}$ a saturação por bases (>70\%). O P disponível foi o mais elevado entre os usos e manejos avaliados, principalmente na camada $0,00-0,10 \mathrm{~m}$. Os teores de $\mathrm{Al}$ trocável $\left(<0,5 \mathrm{cmol}_{\mathrm{c}} \mathrm{dm}^{-3}\right)$ e de saturação por $\mathrm{Al}(<8 \%)$ diminuíram expressivamente em relação ao solo sob $\mathrm{CN}$. A redução de K no LA ocorreu, possivelmente, pela elevada demanda desse elemento pela cultura do milho, bem como por sua exportação na silagem.

Com base nas correlações entre os atributos analisados nas cinco camadas do solo e nos diferentes usos e manejos avaliados, verificou-se que a maioria dos atributos químicos do solo foram condicionados, principalmente, por variações nos teores de COT e no pH do solo (Tabela 4). Isso evidencia a importância da matéria orgânica no comportamento químico dos solos nos CCS, bem como do entendimento de sua dinâmica em resposta às mudanças das condições originais deles.

Área superficial específica (ASE) e capacidade máxima de adsorção de fósforo (CMAP)

A ASE do solo na camada de $0,00-0,05 \mathrm{~m}$ decresceu na sequência $\mathrm{MN}>\mathrm{CNq}>\mathrm{LA} \sim \mathrm{FP}>\mathrm{CN}$, com valores, respectivamente, de 69,1; 59,1; 55,2; 54,9; e $52,9 \mathrm{~m}^{2} \mathrm{~g}^{-1}$. A ASE apresentou relação positiva com o COT no solo sob os distintos usos e manejos (ASE $=0,247 * \mathrm{COT}+46,7 ; \mathrm{r}=0,858 ; \mathrm{P}<0,0001)$. Essa relação contraria aquelas verificadas em estudos em que a ASE foi estimada por adsorção de $\mathrm{N}_{2}$ (AFIF et al., 1995; ALMEIDA et al., 2003), possivelmente, devido a um efeito restritivo da MOS ao contato $\mathrm{N}_{2}$-superfície mineral durante o processo de adsorção. No método de adsorção de água, utilizado no presente estudo, além de não restringir o contato molécula de $\mathrm{H}_{2} \mathrm{O}$ superfície mineral, a MOS também participa como adsorvente de água.

A CMAP do solo decresceu na sequência $\mathrm{FP}>\mathrm{CNq}>\mathrm{CN} \sim \mathrm{LA} \sim \mathrm{MN}$, com valores, respectivamente, de $3.500,3.014,2.459,2.441$ e $2.361 \mathrm{mg} \mathrm{kg}^{-1}$. A magnitude de adsorção de $\mathrm{P}$ (CMAP>2.300 $\mathrm{mg} \mathrm{kg}^{-1}$ ) decorreu da mineralogia caulinítica e oxídica, característica de solos em avançado estágio de intemperização (FONTES \& WEED, 1996; ALMEIDA et al., 2003). Nesse sentido, parece ser contraditória a diminuição da CMAP com o aumento da ASE do solo sob os diferentes usos e manejos avaliados $(\mathrm{CMAP}=-64,887 * \mathrm{ASE}+6625$; $\mathrm{r}=0,569 ; \mathrm{P}=0,0270)$. Entretanto, da mesma forma que pode ter superestimado os valores de ASE do solo, o aumento de COT determinou uma diminuição na CMAP (CMAP $=-22,447 * \mathrm{COT}+3891 ; \mathrm{r}=0,683 ; \mathrm{P}=0,0051)$. Esse comportamento pode ser atribuído tanto ao bloqueio das superfícies minerais, como discutido anteriormente para o $\mathrm{N}_{2}$ na estimativa da ASE, como pela ocupação dos sítios de adsorção pelos grupos funcionais da MOS (FONTES et al., 1992; RHEINHEIMER et al., 2003).

\section{CONCLUSÃO}

Com relação ao campo nativo, a mata nativa apresentou teores de $\mathrm{C}$ orgânico total maiores na camada $0-0,05 \mathrm{~m}$ do solo, enquanto os usos florestamento de Pinus e a lavoura reduziram os teores de $\mathrm{C}$ orgânico total. No manejo do campo com queima da pastagem, os níveis de $\mathrm{C}$ orgânico no solo foram equivalentes aos do campo nativo. $\mathrm{O}$ solo sob campo nativo apresenta baixos níveis de fertilidade, os quais se alteram positivamente sob mata nativa, devido à biociclagem; e pelo manejo recente (sete dias) do campo com a queima da pastagem, através da formação de cinzas contendo cátions básicos e fósforo. A fertilidade do solo foi reduzida sob o uso florestamento de pinus, com aumentos nos teores de alumínio trocável e de saturação por alumínio. Os atributos químicos do solo sob os diferentes usos e manejo do solo avaliados são condicionados pelo conteúdo de matéria orgânica do solo. Os teores de C orgânico total relacionam-se positivamente com a área superficial específica e negativamente com a capacidade máxima de adsorção de fósforo do solo.

Tabela 4 - Valores de "r" e "P" das análises de correlações lineares do COT e pH em $\mathrm{H}_{2} \mathrm{O}$ com os atributos químicos soma de bases (S), saturação por bases $(\mathrm{V})$, saturação por alumínio $(\mathrm{m})$, cálcio $(\mathrm{Ca})$, capacidade de troca de cátions $\left(\mathrm{CTC}_{\mathrm{pH} 7}\right)$, magnésio $(\mathrm{Mg})$, alumínio trocável $(\mathrm{Al})$, potássio $(\mathrm{K})$ e fósforo $(\mathrm{P})$.

\begin{tabular}{lllllllllllll}
\hline & & $\mathrm{pH}$ & $\mathrm{Ca}$ & $\mathrm{Mg}$ & $\mathrm{K}$ & $\mathrm{S}$ & $\mathrm{Al}$ & $\mathrm{CTC}_{\mathrm{pH} 7}$ & $\mathrm{~V}$ & $\mathrm{~m}$ & $\mathrm{P}$ \\
\hline $\mathrm{COT}$ & $\mathrm{r}$ & 0,0561 & 0,6827 & 0,6025 & 0,6180 & 0,7081 & $-0,3854$ & 0,4241 & 0,4598 & $-0,4764$ & 0,3617 \\
& $\mathrm{P}$ & 0,6055 & $<0,0001$ & $<0,0001$ & $<0,0001$ & $<0,0001$ & 0,0002 & $<0,0001$ & $<0,0001$ & $<0,0001$ & 0,0006 \\
& & & & & & & & & & & \\
$\mathrm{pH}$ & $\mathrm{r}$ & 1,0000 & 0,4635 & 0,7191 & 0,0333 & 0,5412 & $-0,8319$ & $-0,5735$ & 0,7644 & $-0,7481$ & 0,4623 \\
& $\mathrm{P}$ & & $<0,0001$ & $<0,0001$ & 0,7593 & $<0,0001$ & $<0,0001$ & $<0,0001$ & $<0,0001$ & $<0,0001$ & $<0,0001$ \\
\hline
\end{tabular}




\section{AGRADECIMENTOS}

Ao Conselho Nacional de Desenvolvimento Científico e Tecnológico (CNPq) pela bolsa de mestrado do primeiro autor (Edital MCT/CNPq no 70/2008 - processo 578100/ 2008-8), pelas bolsas de produtividade em pesquisa de INDA, A.V. e DICK, D.P., e pelo suporte financeiro (Edital Universal2009: proc. 470718/2009-9). Ao professor PhD. Aino Victor Ávila Jacques, por ceder sua propriedade para a realização do estudo e pelas informações sobre o histórico das áreas.

\section{REFERÊNCIAS}

AFIF, E. et al. Organic matter delays but does not prevent phosphate sorption by Cerrado soils from Brazil. Soil Science, v.159, n.3, p.207-211, 1995. Disponível em: <http:// journals.1ww.com/soilsci/Abstract/1995/03000/ Organic_Matter_Delays_But_Does_Not_Prevent.8.aspx $>$. Acesso em: 02 abr. 2012.

ALMEIDA, J.A. et al. Cor do solo, formas do fósforo e adsorção de fosfatos em Latossolos desenvolvidos de basalto do extremosul do Brasil. Revista Brasileira de Ciência do Solo, v.27, n.6, p.985-1002, 2003. Disponível em: <http://www.scielo.br/ scielo.php? script $=$ sci_arttext \& pid = S $0100-$ $06832003000600003 \& \operatorname{lng}=$ pt\&nrm=iso $>$. Acesso em: 02 abr. 2012. doi: 10.1590/S0100-06832003000600003.

CARVALHO, A.M.X. et al. Atividade microbiana de solo e serapilheira em áreas povoadas com Pinus elliottii e Terminalia ivorensis. Revista Brasileira de Ciência do Solo, v.32, número especial, p.2707-2716, 2008. Disponível em: <http:/ /www.scielo.br/scielo.php?script=sci_arttext\&pid=S0100$06832008000700014 \& \operatorname{lng}=$ pt\&nrm=iso $>$. Acesso em: $15 \mathrm{dez}$. 2011. doi: 10.1590/S0100-06832008000700014.

DICK. D.P. et al. Impacto da queima nos atributos químicos e na composição química da matéria orgânica do solo e na vegetação. Pesquisa Agropecuária Brasileira, v.43, n.5, p.633-640, 2008. Disponível em: <http://www.scielo.br/ scielo.php? script=sci_arttext\&pid=S 0100 204X2008000500011\&lng=pt\&nrm=iso>. Acesso em: $15 \mathrm{dez}$. 2011. doi: 10.1590/S0100-204X2008000500011.

DICK, D. P. et al. Pinus afforestation in South Brazilian highlands: soil chemical attributes and organic matter composition. Scientia Agricola, v.68, n.2, p.175-181, 2011. Disponível em: <http:// www.scielo.br/scielo.php? script $=$ sci_arttext\&pid=S0103$90162011000200007 \& \operatorname{lng}=p t \& n r m=i s o>$. Acesso em: $15 \mathrm{dez}$. 2011. doi: 10.1590/S0103-90162011000200007.

FONTES, M.R. et al. Association of microcrystalline goethite and humic acid in some Oxisols from Brazil. Soil Science Society of America Journal, v.56, n.3, p.982-990, 1992. Disponível em: <https://www.soils.org/publications/sssaj/ abstracts/56/3/SS0560030982>. Acesso em: 02 abr. 2012. doi: 10.2136/sssaj1992.03615995005600030050x.

FONTES, M.P.F.; WEED, S.B. Phosphate adsorption by clays from Brazilian Oxisols: relationships with specific area and mineralogy. Geoderma, v.72, p.37-51, 1996. Disponível em: <http://www.sciencedirect.com/science/article/pii/ 0016706196000109>. Acesso em: 02 abr. 2012.
HERINGER, I. et al. Características de um Latossolo Vermelho sob pastagem natural sujeita à ação prolongada do fogo e de práticas alternativas. Ciência Rural, v.32, n.2, p.309-314, 2002. Disponível em: <http://www.scielo.br/scielo.php?script=sci_arttext\&pid=S0103$84782002000200021 \& \operatorname{lng}=$ pt\&nrm=iso >. Acesso em: 15 dez. 2011. doi: 10.1590/S0103-84782002000200021.

HERINGER, I.; JACQUES, A.V.A. Acumulação de forragem e material morto em pastagem nativa sob distintas alternativas de manejo em relação às queimadas. Revista Brasileira de Zootecnia, v.31, n.2, p.599-604, 2002. Disponível em: <http:/ $/ \mathrm{www}$. scielo.br/scielo.php? script $=$ sci_arttext\&pid $=\mathrm{S} 1516$ $35982002000300009 \& \operatorname{lng}=$ pt\&nrm=iso $>$. Acesso em: $15 \mathrm{dez}$. 2011. doi: $10.1590 / \mathrm{S} 1516-35982002000300009$.

HERINGER, I.; JACQUES, A.V.A. Qualidade da forragem de pastagem nativa sob distintas alternativas de manejo. Pesquisa Agropecuária Brasileira, v.37, n.3, p.399-406, 2002. Disponível em: <http:// www.scielo.br/scielo.php? script $=$ sci_arttext\&pid $=S 0100$ 204X2002000300022\&lng=pt\&nrm=iso>. Acesso em: 15 dez. 2011. doi: 10.1590/S0100-204X2002000300022.

KÄMPF, N.; SCHWERTMANN, U. The 5-M- $\mathrm{NaOH}$ concentration treatment for iron oxides in soils. Clays and Clay Minerals, v.30, p.401-408, 1982.

LA TORRACA, S.M. et al. Recrutamento e exportação de nutrientes por Pinus elliottii var. elliottii em Latossolo VermelhoEscuro na região de Agudos, SP. IPEF, n.27, p.41-47, 1984.

MAFRA, A.L. et al. Carbono orgânico e atributos químicos do solo em áreas florestais. Revista Árvore, v.32, n.2, p.217-224, 2008. Disponível em: <http://www.scielo.br/scielo.php?script=sci_arttext\&pid=S010067622008000200004\&lng=en\&nrm=iso>. Acesso em: 15 dez. 2011. doi: 10.1590/S0100-67622008000200004.

MEHRA, O.P.; JACKSON, M.L. Iron oxide removal from soils and clays by a dithionite-citrate system buffered with sodium bicarbonate. Proceedings Clays and Clay Mineral Conference, v.7, p.317-327, 1960.

MURPHY, J.; RILEY, J.P. A modified single solution method for the determination of phosphate in natural waters. Analytica Chimica Acta, v.27, p.31-36, 1962. Disponível em: <http:// www.sciencedirect.com/science/article/pii/ S0003267000884445>. Acesso em: 02 abr. 2012. doi:10.1016/ S0003-2670(00)88444-5.

OVERBECK, G.E. et al. Brazil's neglected biome: The South Brazilian Campos. Perspectives in Plant Ecology, Evolution and Systematics, v.9, p.101-116, 2007. Disponível em: <http://www.sciencedirect.com/science/article/pii/ S1433831907000303>. Acesso em: 02 abr. 2012. doi: 10.1016/ j.ppees.2007.07.005.

PILLAR, V.P.; QUADROS, F.L.F. Grassland-forest boundaries in southern Brazil. Coenoses, v.12, p.119-126, 1997.

QUIRK, J.P. Significance of surface areas calculated from water vapour sorption isotherms by the use of the BET equation. Soil Science, v.80, n.6, p.423-430, 1955. Disponível em: <http://journals.1ww.com/soilsci/Citation/1955/12000/ Significance_of_Surface_Areas_Calculated_From.1.aspx $>$. Acesso em: 02 abr. 2012. 
RHEINHEIMER, D.S. et al. Sorção de fósforo em função do teor inicial e de sistemas de manejo de solos. Revista Brasileira de Ciência do Solo, v.27, n.1, p.41-49, 2003a.

RHEINHEIMER, D.S. et al. Modificações nos atributos químicos de solo sob campo nativo submetido à queima. Ciência Rural, v.33, n.1, p.49-55, 2003b. Disponível em: <http://www.scielo.br/ scielo.php?script=sci_arttext\&pid=S 0103 $84782003000100008 \& \operatorname{lng}=$ pt\&nrm=iso $>$. Acesso em: $15 \mathrm{dez}$. 2011. doi 10.1590/S0103-84782003000100008.
STRECK, E. V. et al. Solos do Rio Grande do Sul. 2.ed. Porto Alegre: Emater/RS-ASCAR, 2008. 222p.

TEDESCO, M.J. et al. Análise de solo, plantas e outros materiais. 2.ed. Porto Alegre: Universidade Federal do Rio Grande do Sul, 1995. 174p. (Boletim Técnico, 5).

WIESMEIER, M. et al. Depletion of soil organic carbon and nitrogen under Pinus taeda plantations in Southern Brazilian grasslands (Campos). European Journal of Soil Science, v.60, n.3, p.347-359, 2009. Disponível em: <http:// on linelibrary.wiley.com/doi/10.1111/j.13652389.2009.01119.x/abstract>. Acesso em: 02 abr. 2012. 